\title{
Ações de extensão sobre a sexualidade e infecções sexualmente transmissíveis aos idosos: relato de experiência
}

\author{
Extension actions on sexuality and sexually transmissible infections to the elderly: \\ experience report
}

\section{Acciones de extensión sobre sexualidad e infecciones de transmisión sexual a personas} mayores: relato de experiencia

Silvana Bastos $\operatorname{Cogo}^{1}$, Laís Mara Caetano da Silva ${ }^{1}$, Lívia Martins de Martins ${ }^{1 *}$, Anna Júlia Pacheco Alves ${ }^{1}$, Anne Louíze Menezes Xavier', Bruna Caroline Ruppelt', Carine Rieger Donel', Elisa Fortes Vilhalba ${ }^{1}$, Hentielle Feksa Lima ${ }^{1}$, Jully Martins Gomes Portela'1.

\section{RESUMO}

Objetivo: Relatar a experiência de acadêmicos de enfermagem, integrantes do Programa de Educação Tutorial (PET) Enfermagem face às ações de extensão desenvolvidas com idosos sobre sexualidade na terceira idade e exposição às Infecções Sexualmente Transmissíveis (ISTs). Relato da experiência: $O$ estudo foi desenvolvido a partir da experiência dos integrantes do PET Enfermagem na implementação de uma oficina direcionada à idosos sobre sexualidade na terceira idade e ISTs. A atividade foi desenvolvida em 2019, em um evento realizado, anualmente, por intermédio de uma universidade federal no interior do Rio Grande do Sul. As ações propostas para abordar a temática, foram divididas em dois momentos: o "Bingo da Sexualidade" e o "Bailão da Sexualidade". As perguntas elaboradas para a dinâmica abordavam as ISTs e sintomas mais prevalentes, exames preventivos disponíveis no SUS, cuidados de higiene íntima e questões relacionadas à autoestima. Considerações finais: As evidências encontradas nas atividades desenvolvidas, demonstram a pertinência da abordagem sobre o tema, visto que há carência de informações para esse público, no que tange aos aspectos da sexualidade e das ISTs. A oficina desenvolvida pôde ser considerada uma ferramenta de educação em saúde capaz de proporcionar aos idosos um ambiente de integração, trocas e construção de conhecimento.

Palavras-chave: Idoso, Doenças sexualmente transmissíveis, Enfermagem, Educação em saúde.

\begin{abstract}
Objective: To report the experience of nursing students, members of the Tutorial Education Program (PET) Nursing in view of the extension actions developed with the elderly about sexuality in the elderly and exposure to Sexually Transmitted Infections (STIs). Experience report: The study was developed based on the experience of the members of PET Nursing in implementing a workshop aimed at the elderly about sexuality in the elderly and STIs. The activity was developed in 2019, in an event held, annually, through a federal university in the interior of Rio Grande do Sul. The actions proposed to address the theme, were divided into two moments: the "Bingo da Sexualidade" and the "Bailão da Sexualidade". The questions elaborated for the dynamics addressed the most prevalent STIs and symptoms, preventive exams available in SUS, intimate hygiene care and issues related to self-esteem. Final considerations: The evidence found in the activities developed, demonstrates the pertinence of the approach on the theme, since there is a lack of information for this public, regarding the aspects of sexuality and STIs. The developed workshop could be considered a health education tool capable of providing the elderly with an environment of integration, exchange and construction of knowledge.
\end{abstract}

Keywords: Elderly, Sexually transmitted diseases, Nursing, Health education.

\footnotetext{
1 Universidade Federal de Santa Maria (UFSM), Programa de Educação Tutorial (PET), Santa Maria - RS
}

*E-mail: liviamartinsm13@gmail.com 


\section{RESUMEN}

Objetivo: Informar la experiencia de los estudiantes de enfermería, integrantes del Programa de Educación Tutorial (PET) Enfermería ante las acciones de extensión desarrolladas con los adultos mayores sobre la sexualidad en los ancianos y la exposición a Infecciones de Transmisión Sexual (ITS). Informe de experiencia: El estudio se desarrolló a partir de la experiencia de los miembros de Enfermería PET en la implementación de un taller dirigido a personas mayores sobre sexualidad en personas mayores e ITS. La actividad se desarrolló en 2019, en un evento que se realiza anualmente a través de una universidad federal en el interior de Rio Grande do Sul. Las acciones propuestas para abordar el tema, se dividieron en dos momentos: el "Bingo da Sexualidade" y el "Bailão da Sexualidade". Las preguntas elaboradas para la dinámica abordaron las ITS y síntomas más prevalentes, exámenes preventivos disponibles en el SUS, cuidados de higiene íntima y temas relacionados con la autoestima. Consideraciones finales: La evidencia encontrada en las actividades desarrolladas, demuestra la pertinencia del enfoque sobre el tema, ya que existe una falta de información para este público, sobre los aspectos de la sexualidad y las ITS. El taller desarrollado podría considerarse una herramienta de educación en salud capaz de brindar a las personas mayores un ambiente de integración, intercambio y construcción de conocimientos.

Palabras clave: Anciano, Enfermedades de transmisión sexual, Enfermería, Educación para la salud.

\section{INTRODUÇÃO}

As Infecções Sexualmente Transmissíveis (ISTs) são causadas por vírus, bactérias, fungos e outros patógenos, transmitidas, principalmente, por meio do contato sexual desprotegido. Ainda, podem ser assintomáticas ou apresentarem sinais e sintomas distintos, que por vezes levam tempo para serem percebidos pela pessoa infectada (BRASIL, 2019).

As ISTs ocorrem com alta frequência na população e uma realidade cada vez mais presente entre as pessoas idosas. No contexto da sexualidade, pesquisadores têm indicado que os idosos continuam sexualmente ativos, inclusive após os 80 anos de idade (ANDRADE J, et al., 2017). Estudos epidemiológicos evidenciam que na população de 50 anos ou mais, a taxa de ISTs aumentou, principalmente na América do Norte, China e África Sub-saariana. No Brasil, ainda não há dados em âmbito nacional acerca da prevalência das ISTs em geral e entre idosos. Além disso, para o HIV tem-se o aumento nas taxas entre homens e mulheres, entre 60 anos ou mais, baseado em dados dos últimos 10 anos, o que indica a vulnerabilidade de idosos à sua ocorrência (ANDRADE J, et al., 2017).

Muitos fatores estão relacionados à vulnerabilidade da população idosa em relação às ISTs. O principal agravante é o idoso ser visto como um ser assexuado pela sociedade, o que gera a dispensa da abordagem preventiva (BRASIL, 2018). Dessa forma, por ser um assunto velado na sociedade e na família, compila para a falta de informação e cuidados que essa população deve ter com sua saúde e sexualidade na fase da velhice. Nesse viés, as ações de educação em saúde e o desenvolvimento de programas relativos às ISTs em idosos, em diferentes contextos, configuram-se como estratégias para o enfrentamento aos tabus, mitos e preconceitos que permeiam a sexualidade na velhice (ROZENDO AS e ALVES JM, 2015).

Logo, as evidências encontradas nas atividades desenvolvidas, demonstram a pertinência da abordagem sobre o tema, em diferentes espaços sociais, visto que ainda há informações inseguras nesse público no que tange aos aspectos das ISTs. Ademais, a experiência citada no presente estudo, realizou-se por meio de um projeto, organizado para um evento realizado anualmente, por intermédio de uma universidade federal localizada no interior o Rio Grande do Sul. O evento propicia a integração entre acadêmicos e idosos ativos, a partir da realização de oficinas e atividades que estimulam o envelhecimento saudável por meio da educação em saúde.

As atividades propostas, relativas à Sexualidade na Terceira Idade, vão ao encontro do proposto pelo evento. Haja vista na vivência efetuada, as ações de extensão apresentam-se como fundamentais à promoção da saúde e prevenção das ISTs. Além disso, produzem impactos satisfatórios nesse público, corroboram a qualidade de vida e possibilitam espaços de discussões e reflexões. 
Para tanto, tem-se por objetivo relatar a experiência dos acadêmicos de enfermagem, integrantes do Programa de Educação Tutorial Enfermagem face às ações de extensão desenvolvidas com os idosos sobre a sexualidade e infecções sexualmente transmissíveis.

\section{RELATO DE EXPERIÊNCIA}

Trata-se de um relato de experiência, a partir de vivências dos acadêmicos integrantes do Programa de Educação Tutorial (PET) Enfermagem de uma universidade federal do interior do Rio Grande do Sul (RS). As atividades realizadas foram direcionadas à idosos ativos, abrangendo as temáticas: sexualidade e ISTs, por meio de um evento destinado a esse público, em uma cidade do interior do RS. O evento é caracterizado pela realização de oficinas ministradas e organizadas por diferentes cursos de graduação (enfermagem, odontologia, fisioterapia, dança, psicologia, educação física, entre outros) com foco nas demandas do públicoalvo.

Na edição do ano de 2019, os integrantes do grupo PET Enfermagem propuseram a temática Sexualidade na terceira idade para guiar as oficinas a serem ministradas. As ações propostas para abordar a temática, foram divididas em dois espaços: o Bingo e o Bailão da Sexualidade. As oficinas, mediadas pelos acadêmicos de enfermagem, iniciaram às 11 horas e se estenderam até às $16 \mathrm{~h} 30 \mathrm{~min}$, atendendo um público de cerca de 160 idosos.

No desenvolvimento da oficina Bingo da Sexualidade, o número de mulheres participantes se manteve superior em relação aos homens. A dinâmica ocorreu com a distribuição de cartelas de bingo e feijões aos participantes, após, a cada número sorteado, os mediadores realizavam uma pergunta aos oficineiros em relação à temática sexualidade na terceira idade. As perguntas tinham o intuito de estimular as discussões acerca da temática, portanto, abordavam, especialmente as ISTs e seus sintomas, exames preventivos disponíveis no SUS, cuidados com a higiene íntima e questões de autoestima.

Neste sentido foi possível estimular a participação das pessoas idosas e mesmo com a experiência proporcionada pelos anos de vida, muitos idosos ainda não possuem informações no que tange ao envelhecimento e sexualidade. No transcorrer das rodadas, que duravam cerca de 30 minutos, a participação e interação das mulheres idosas se mostrou mais efetiva que a dos homens.

$\mathrm{Na}$ oficina Bailão da Sexualidade, começou com os mediadores solicitando que os oficineiros formassem duplas para que dançassem enquanto as músicas tocavam e quando eram pausadas, o mediador retirava de uma caixinha, afirmações sobre ISTs e sexualidade e em seguida lia para todos em voz alta. O intuito das afirmações era de instigar a reflexão sobre cada uma delas e promover uma discussão para que, posteriormente respondessem acerca de cada afirmativa, se consideravam verdadeira ou falsa. Após ouvirem as respostas dos participantes, os mediadores explicavam o porquê da afirmação, além de sanar dúvidas e discutir com os demais cada questão elencada.

Os idosos participaram atentamente da atividade proposta, colaborando com a discussão, pois comentavam a respeito das vivências pessoais. Além disso, trouxeram mitos e verdades, além das afirmações preparadas para a atividade, o que enriqueceu a proposta. A avaliação da atividade se deu de maneira informal, por meio dos relatos positivos proferidos pelos participantes após o término das atividades. Ainda, ao sair da sala, escreveram carinhosos recados de agradecimento em um mural disponibilizado pelos acadêmicos.

Os acadêmicos integrantes do PET Enfermagem obtiveram resultados satisfatórios com a realização da atividade, observados por meio da avaliação positiva dos idosos e da conclusão do grupo sobre o quão comum é, encontrar idosos que não têm acesso a informações corretas acerca das temáticas discutidas, o que implica na forma como cuidam da saúde nessa etapa da vida.

\section{DISCUSSÃO}

Os estereótipos de uma velhice assexuada perpetuam-se culturalmente e são presentes no imaginário social. A velhice na cultura ocidental é sinônimo de incapacidade, decadência, perdas biológicas e sociais, e 
inatividade sexual (ROZENDO AS e ALVES JM, 2015). A abordagem da sexualidade na terceira idade vem acompanhada de preconceitos, mitos e tabus, a partir da crença de que o processo de envelhecimento impossibilita o idoso de manter uma vida sexual ativa.

Perante a sociedade, o idoso é visto como um ser assexuado, cuja prática da sexualidade tornou-se incomum, o que pode gerar uma desatenção por parte dos profissionais de saúde e familiares, aumentando a vulnerabilidade dessa população (BRASIL, 2018a). Durante a oficina, elucidou-se o preconceito da família em relação à sexualidade na terceira idade. Sob essa questão, pontua-se que a sexualidade está intrínseca nas diferentes etapas da vida de homens e mulheres, incluindo jovens, adultos e idosos e é entendida como um componente essencial da existência humana, que varia de acordo com o contexto social, cultural e religioso Entende-se por sexualidade a relação que vai além do contato físico e corporal, visto que existe uma junção de prazeres envolvidos na sexualidade: harmonia do casal, afeto, aspectos culturais e sociais (VIEIRA KFL, et al., 2016).

Estudos mostram que $74 \%$ dos homens e $56 \%$ das mulheres casadas mantêm vida sexual ativa após os 60 anos. Muitas das disfunções e alterações sexuais que ocorrem com o avançar da idade podem ser resolvidas com escuta, orientação e educação, pois podem ser provocadas por problemas psicológicos, fisiológicos ou ambos (CUNHA LM, et al., 2015).

Para tanto, o MS orienta sobre a importância de abordar as questões sexuais da população idosa, por fatores como: o crescente envelhecimento populacional dos brasileiros, o aumento das ISTs entre indivíduos com mais de 60 anos, e também por se tratar de um aspecto fundamental para a qualidade de vida dessas pessoas (BRASIL, 2018a).

Nas oficinas com os idosos percebeu-se, que embora os idosos fossem ativos, saudáveis e com a capacidade cognitiva preservada, ainda apresentaram desconhecimento sobre algumas das ISTs. Os idosos que ultrapassam as barreiras relacionadas ao envelhecimento e vivem uma sexualidade satisfatória e saudável são aqueles que recebem uma assistência acolhedora dos profissionais de saúde. Para isso, os profissionais e acadêmicos de enfermagem devem isentar-se de preconceitos, preparados para fornecer suporte aos idosos acerca das modificações envolvidas na plasticidade do corpo, nos fatores biológicos que interferem na sexualidade e na prevenção das ISTs (CASTRO SFF, et al., 2013).

Além disso, embora as taxas masculinas sejam significativas nos perfis de morbimortalidade, observa-se que a presença do sexo masculino nos serviços de saúde é menor que a das mulheres. Além de não procurarem os serviços de saúde, alguns dados epidemiológicos demonstram que o público masculino possui hábitos inadequados quanto a alimentação, atividades físicas, uso do álcool e outras drogas e mais suscetível à ISTs e Aids (Síndrome de Imunodeficiência Adquirida) (BRASIL, 2018b). Ainda, historicamente há questões culturais na forma como o homem é visto na sociedade, forte, viril, chefe de família e dessa forma, o cuidado com saúde não é reconhecido por muitos indivíduos, como uma prática masculina (BARROS CT, et al., 2018).

Diante disso, os resultados em relação ao número de homens compondo o público idoso presente nas oficinas desenvolvidas, parecem ir ao encontro das informações apresentadas. Também, demonstram que há pouca adesão dos homens, não somente nos serviços de saúde, mas nos espaços em que a discussão permeia as questões de cuidados com a saúde. Tem-se o fato de que há um menor número de idosos homens, o que contribui para a menor participação desse público nas oficinas. O homem idoso acaba por negligenciar, as consultas, a realização de exames do aparelho reprodutor masculino, a realização de teste rápido, importantes na prevenção e diagnóstico de doenças, como o câncer de próstata e ISTs (ANDRADE J, et al., 2017).

O público feminino, embora apresente vulnerabilidades em relação às questões de saúde sexual e reprodutiva, realiza consultas ginecológicas de forma periódica devido ao conhecimento acerca das mudanças fisiológicas, exame preventivo, teste rápido, além de buscar acolhimento nos serviços de saúde. A mulher idosa tem acesso a informações acerca dos cuidados em relação às mudanças proporcionadas pelo envelhecimento, de como pode prevenir doenças e como manter uma vida sexual saudável (COSTA E SILVA SP e MENANDRO MCC, 2014). 
Nessa perspectiva, apesar da Política Nacional de Atenção Integral à Saúde do Homem (PNAISH), sensibilizar os profissionais da saúde e os homens, quanto à necessidade de estes buscarem os serviços da rede SUS, é necessário que essa questão seja abordada em outros espaços. Com isso, incentivar e informar os públicos na terceira idade, acerca da temática se torna fundamental para melhores resultados, principalmente em relação ao público masculino, sejam atingidos (BRASIL, 2018b).

Por meio das oficinas, percebeu-se que muitos idosos não possuem informações seguras no que se refere à sexualidade e o envelhecimento, o que evidencia a persistência de mitos acerca da temática. O preconceito existente corrobora o baixo conhecimento dos idosos a respeito do assunto, uma vez que se torna vergonhoso e/ou constrangedor falar sobre, tornando esse público mais suscetível à ISTs.

Além disso, segundo pesquisas, outro fator que influencia esta situação, é a informação limitada na juventude, em maioria, conhecimentos populares da época, no que tange aos aspectos do uso de preservativo e métodos para prevenção de ISTs e Aids (UCHÔA YS, et al., 2016). Nesse viés, sabe-se que idosos com pouco conhecimento em relação à sexualidade e aos cuidados, estão mais suscetíveis à ISTs (AGUIAR RB, et al., 2020). Com isso, os serviços de saúde e os profissionais devem contribuir à propagação de informações seguras sobre envelhecimento, sexualidade. Ademais, a relação e interação dos profissionais para com os idosos, paralelo a atividades educativas, contribui para o aumento da adesão aos serviços, o que resulta, principalmente, à atenção integral do indivíduo e possibilita a elucidação de dúvidas, maior conhecimento sobre a sexualidade e os cuidados (ALENCAR DL, et al., 2014).

Nesse sentido, embora evidente a relação do envelhecimento e a vulnerabilidade à ISTs, ainda há poucas investigações, no Brasil, que abordam os fatores associados à problemática (ANDRADE J, et al., 2017). Dessa forma, apoiar e explanar o assunto em diferentes meios sociais corrobora e facilita o acesso dos idosos aos serviços de saúde, resultando em maiores informações e a redução da vulnerabilidade existente. Assim, a sexualidade do idoso deve ser compreendida a partir do princípio de que ela faz parte da totalidade do indivíduo, portanto, um fator biopsicossociocultural, e não apenas biológico (ALENCAR DL, et al., 2014).

Neste contexto, leva-se em consideração os estudos citados anteriormente, que indicam que o processo de envelhecimento, quando privado de informações relacionados a saúde sexual, pode associar-se ao estado de vulnerabilidade dos idosos em contrair ISTs. Por isso, salienta-se a importância de organizar ações educativas em saúde que permitam que os idosos discutam e reflitam sobre informações e questões relacionadas à sexualidade que implicam nessa etapa da vida (BARRETO RMA, et al., 2016).

As ações de educação em saúde podem ser consideradas ferramentas que colaboram para o estímulo do desenvolvimento de pensamento crítico a determinado assunto, no caso da atividade, a sexualidade e ISTs em idosos.Também favorece a autonomia e autocuidado, possibilitando ao público idoso, a participação de discussões referentes às questões da atividade. Ainda, auxilia na percepção dos seus próprios costumes e hábitos, que podem influenciar de maneiras diversas e significativas a sua saúde (SILVA WB, et al., 2019).

Além disso, atividades lúdicas permitem a socialização do grupo participante juntamente com os acadêmicos integrantes do PET. Com isso, promove de forma articulada o exercício cognitivo para participação da atividade do Bingo e da Dança, mas também o raciocínio reflexivo para responder corretamente às questões relacionadas à sexualidade e às ISTs e discuti-las juntamente com o grupo, promovendo a detecção da importância do autoconhecimento e autopercepção sobre o corpo e saúde sexual (BARROS EJL, et al., 2012 ;CYRINO RS, et al., 2016; OLIVEIRA FA, et al., 2018).

As oficinas desenvolvidas pelos acadêmicos integrantes do PET sobre sexualidade e ISTs na terceira idade, configuram-se como uma ferramenta de educação em saúde capaz de proporcionar aos idosos um ambiente de integração, autocuidado, autonomia e aquisição de conhecimentos acerca da sexualidade, além de trocas de experiências compartilhadas com o grupo. Ainda, possibilitou aos acadêmicos a aproximação com idosos e a reflexão sobre a necessidade da elaboração de atividades lúdicas que atraiam sua participação e permitam a difusão de informações importantes para essa fase da vida, dentro do contexto da sexualidade e ISTs. A atividade permitiu que o processo de formação em enfermagem seja aprimorado, de modo que esta oportunidade propicie aos acadêmicos, o exercício de seus conhecimentos, o aprimoramento do senso crítico e habilidades como futuros profissionais capazes de atuar nos âmbitos da assistência e da gestão, articulando ensino e promoção da saúde. 


\section{REFERÊNCIAS}

1. AGUIAR RB, et al. Conhecimento e atitudes sobre sexualidade em pessoas idosas com HIV. Ciência \& Saúde Coletiva, 2020; 25(6): 2051-2062.

2. ALENCAR DL, et al. Fatores que interferem na sexualidade de idosos: uma revisão integrativa. Ciência e Saúde Coletiva, 2014; 19(8): 3533-3542.

3. ANDRADE J, et al. Vulnerabilidade de idosos a infecções sexualmente transmissíveis. Acta Paul Enferm, 2017; 30(1): 8-15.

4. BARROS CT, et al. Mas se o homem cuidar da saúde fica meio que paradoxal ao trabalho": relação entre masculinidades e cuidado à saúde para homens jovens em formação profissional. Saúde e Sociedade. Brasil, 2018.

5. BARROS EJL, et al. Gerontotecnologia educativa voltada ao idoso estomizado à luz da complexidade. Rev. Gaúcha Enferm, Porto Alegre, 2012; 33(2).

6. BARRETO RMA, et al. Ações educativas em saúde para o público adolescente: uma revisão integrativa. Rev. APS, 2016; 19(2): 277-285.

7. CASTRO SFF, et al. Sexualidade na Terceira Idade - A percepção do enfermeiro da estratégia saúde da família. Revista de Enfermagem UFPE On Line, 2013; 7(10): 5907-5914.

8. COSTA E SILVA SP, MENANDRO MCC. As representações sociais da saúde e de seus cuidados para homens e mulheres idosos. Saúde Soc, São Paulo, 2014; 23(2): 626-640.

9. CUNHA LM, et al. Vovó e vovô também amam: sexualidade na terceira idade. Rev. Mineira de Enfermagem, 2015.

10. CYRINO RS, et al. Atividades lúdicas como estratégia de educação em saúde com

11. idosos. Rev. Ciência em extensão,2016; 12(3): 154-163.

12. MINISTÉRIO DA SAÚDE. Protocolo Clínico e diretrizes terapêuticas para atenção integral às pessoas com infecções sexualmente transmissíveis (ISTs). Brasília-DF, 2019.

13. MINISTÉRIO DA SAÚDE. Secretaria de Atenção à Saúde: Saúde do homem. Brasília-DF, 2018b.

14. MINISTÉRIO DA SAÚDE. Secretaria de Atenção Básica, Departamento de Atenção Básica: Saúde sexual e saúde reprodutiva. Brasília-DF, 2018a.

15. OLIVEIRA FA, et al. Atividades de educação em saúde realizadas com grupo de idosas para promoção do autocuidado em saúde. Rev. Eletrônica de Extensão,2018; 15(28): 137-150.

16. OLIVEIRA MM, et al. A saúde do homem em questão: busca por atendimento na atenção básica de saúde. Ciência \& Saúde Coletiva, 2015; 20(1):273-278.

17. ROZENDO AS, ALVES JM. Sexualidade na terceira idade: tabus e realidade, 2015; 18(3): 95-107.

18. SECRETARIA DE ESTADO DA SAÚDE DO PARANÁ (SESA). Superintendência de Atenção à Saúde: Avaliação multidimensional do idoso. Curitiba-PR, 2017.

19. SILVA WB, et al. Educação em saúde acerca da prevenção da violência obstétrica: relato de experiência. Rev. Eletrônica Acervo Saúde, 2019; 11(14).

20. UCHÔA YS, et al. A sexualidade sob o olhar da pessoa idosa. Rev. Bras. Geriatr. Gerontol, Rio de Janeiro, 2016; 19(6): 939-949.

21. VIEIRA KFL, et al. Representação social das relações sexuais: um estudo transgeracional entre mulheres. Psicologia: Ciência e Profissão, 2016; 36(2): 329-340. 